\title{
LAS LENGUAS DORASQUE Y CHANGUENA Y SUS RELACIONES GENEALOGICAS (1)
}

\author{
Adolfo Constenla Umaña
}

\begin{abstract}
The purpose of this article is to examine, by means of methods of historical linguistics other than inspection, the relationships of four extinct indigenous linguistic varieties of Western Panama (Changuena, Chumulu, Gualaca, Dorace) with each other and with a series of Chibchan languages of Costa Rica, Panama, and Colombia.

It is concluded that the four varieties belong to two languages: Dorasque (with two dialects: Gualaca and Chumulu-Dorace) and Changuena, and that both, in turn, constitute an independent branch of the Chibchan stock.
\end{abstract}

0. Introducción

\subsection{Antecedentes históricos}

Los dorasques y los chánguenas fueron dos grupos indígenas importantes del occidente de Panamá. De acuerdo con Pinart (1884, p. 1) la "nación Dorasque-Changuina, en los tiempos de la conquista, tenía su residencia y asiento principal detrás del Volcán de Chiriquí... en las altísimas sierras de Chiriqui y de la Talamanca, donde confinaban con las naciones de la Talamanca (Teribis, Terrabas, Cabecares). Llenaban hasta las costas del mar del norte y laguna de Chiriqui..."

En el siglo XVIII, estos indígenas fueron trasladados en su mayoría al lado del Pacífico y establecidos (ibidem, p. 2) "en las misiones de San José de Bugaba, San Miguel del Boquerón, San Francisco de Dolega y Gualaca", sitios en los que para 1884 ya estaban a punto de extinguirse (ibidem): "en las cercanías de Bugaba... viven unos tres o cuatro Indios de nación Changuina. En la Caldera y El Potrero de Vargas viven unos seis Indios de nación Chumulu. En Gualaca murió en 1882 el ultimo Indio, de la nacion Dorasque propia".

(1) Este artículo, que dedico a la ilustre educadora panameña doña Beatriz Miranda de Cabal, se presentó como ponencia en el Congreso en Honor a Pedro Rómulo Gallegos y a Pedro Henríquez Ureña celebrado en la ciudad de David, Panamá, del 23 al 28 de noviembre de 1984 .
No obstante, la lengua dorasque subsistió en algún grado hasta el siglo XX (Torres de Araúz, 1980 , p. 85 ): "Se tiene noticias de supervivencias de Doraces... hasta bien entrado el presente siglo; autores como Yorke Bell, en 1909 los mencionan como un grupo definido aunque casi extinto. En la primera mitad de este siglo todavía fue posible recoger, un extenso vocabulario Dorasque...".

Según Pinart (ibidem, p. 2), en la zona originaria quedó un grupo, el cháliva o sáliva, que a fines del siglo pasado se decía que existía en un valle aislado del alto Changuinola. De este grupo parece no haber noticias posteriores, aunque el nombre ha seguido mencionándose (o, quizás mejor, repitiéndose). Voegelin (1965, p. 29) afirmó hace 19 años que el cháliva no estaba extinto y que además se hablaban dos dialectos o lenguas muy relacionadas más: el duy y el burica (el segundo de los cuales según él tenía 700 hablantes en Costa Rica y Panamá), sobre las que no parece haber otra referencia. En este trabajo, como ni el cháliva ni el duy ni el burica han sido atestiguados (no parece haberse recogido ni una palabra de ellos), no se tomarán en cuenta. Lo que es más, el hecho de que Torres de Araúz (1980) no haga referencia a los dorasques sino como grupo extinto hace pensar que en 1965 ya no existieran tales grupos (que, de todos modos, bien podrían no haberse dado nunca). En noviembre de 1984 recorrí Dolega y sus alrededores en busca de alguien que supiera algo de dorasque y no pude hallar a nadie. Todas las personas interrogadas parecían estar de acuerdo en que el último conocedor de la lengua había sido el Sr. Reyes Samudio, fallecido varios años atrás. 


\subsection{Materiales sobre las dos lenguas}

Los materiales de las dos lenguas que llegaron a recogerse están contenidos en dos obras: Vocabulario castellano-dorasque: dialectos chumulu, gualaca y chánguina de A. L. Pinart (1884) y Un pueblo visto a través de su lenguaje de B. Miranda de Cabal (1974).

La primera incluye datos de dos formas del dorasque (las hablas de Chumulu y Gualaca) y del chánguena. Los de esta última lengua son los únicos que de ella se han conservado y son también los más escasos: 134 formas en total. Los del chumulu y el gualaca son mucho más abundantes: 431 y 421 rubros respectivamente. Los vocabularios del chumulu y el chánguena fueron recogidos por Pinart. El de gualaca fue tomado por éste de la obra manuscrita del padre Franco (del siglo XVIII) y revisado con el "ultimo Indio de la mision".

La segunda obra incluye un vocabulario mucho más extenso que los de Pinart (aproximadamente 700 palabras y 500 frases cortas) de una forma del dorasque que denominaré dorace, como la autora de la obra, y que, se demostrará más adelante, muestra mayor relación con los datos del habla de Chumulu que con los de habla de Gualaca.

Ambas obras, sin menoscabo de su enorme valor, presentan los problemas habituales de los trabajos hechos sin la ayuda de los métodos de la lingü ística moderna: una transcripción problemática, errores en la identificación de los significados, inclusión de formas complejas (a veces oraciones enteras) como rubros simples de vodabulario, etc. Pero los materiales más difíciles de utilizar son, sin duda, los de Miranda de Cabal, en los que a las dificultades ya mencionadas se suma el hecho de que la persona que se empleó como informante no fue una hablante fluida sino más bien una semihablante, de modo que una buena parte de los rubros incluidos son secuencias de palabras o elementos de tipo onomatopéyico (quizás ya existentes muchos de ellos como ideófonos en la lengua) con que trató de sustituir los elementos léxicos precisos ya olvidados, como se puede apreciar en los siguientes ejemplos:

$\begin{array}{lllll}\text { cocrí } & \text { coolé } & \text { coré } & & \text { 'huevo' } \\ \text { gallina } & \text { hueso } & \text { excremento } & & \\ \text { yi } & \mathrm{b}^{\mathrm{a}} & \mathrm{cab} & \text { güescal 'caldo' } \\ \text { agua } & \text { de } & \text { comida } & \text { delgada } \\ \text { irga } & \text { táguti } & \text { duquí } & \text { balú 'sublevación' } \\ \text { corazón } & \text { alzar } & \text { indio } & \text { jefe } & \end{array}$

$\begin{array}{lcc}\text { sergua } & \text { birish birish } & \text { 'correr' } \\ \text { pie } & \text { (onomatopeya) } & \\ \text { magn } & \text { magn } & \text { 'abanico' } \\ \text { shuip } & \text { shuip } & \text { 'chupar' }\end{array}$

La comparación de las frases contenidas en una y otra obra revelan también que la informante de Miranda de Cabal no sólo desconocía numerosos rubros léxicos, sino que no había adquirido bien la sintaxis de la lengua. La impresión general que producen los datos es, de todos modos, la de estar ante un tipo de habla sumamente simplificado $y$ desviante en relación con la tipología de las lenguas del área. Un claro ejemplo de esto es el uso de un único elemento para relacionar sintagmas nominales, la preposición $b^{\text {a }}$ (para la que la autora del estudio da los siguientes significados: 'para, en, por, hacia, con'), frente a la expresión, en todas las lenguas circundantes de dichas relaciones por medio de una larga serie de posposiciones. En Pinart, en cambio, aunque los ejemplos de frases son muy escasos, cuando se presenta algún relator de sintagmas nominales, se trata de una posposición, como en sivó báliga caga traducido como 'gente que viene por mar' quizás mejor 'el extranjero vino por mar') en que báliga 'por mar' contiene el elemento nominal bali 'mar' y la posposición -ga, que deriva de una posposición protochibcha * ka 'en, hacia' con claros reflejos en un buen número de lenguas (guatuso ko, cabécar $k a$, bocotá ge, cuna ka, muisca ca, cágaba $k a$, boruca $k a$ ).

Por las razones apuntadas, en este estudio, que corresponde a una etapa inicial de las investigaciones que estoy llevando a cabo sobre la lengua, daré preferencia a los datos de Pinart sobre los de Miranda de Cabal, sin que esto implique que los segundos sean menos valiosos; por lo contrario, su interés es enorme desde todo punto de vista; simplemente requieren, para poder aprovecharse plenamente, un grado mayor de análisis del que he realizado hasta el momento y, debido a los fenómenos de simplificación, resultan menos convenientes para hacer comparaciones destinadas a determinar grados de afinidad.

\subsection{Clasificaciones del dorasque y el chánguena}

Pinart (op. cit.) fue al parecer el primero en reconocer la afinidad del chánguena con las hablas de Chumulu y Gualaca. El título mismo de su obra "Vocabulario castellano-dorasque: dialectos 
chumulu, gualaca y chánguina" da a entender que consideraba a las tres hablas mencionadas como variedades de una misma lengua. Esta percepción de la relación existente entre ellas, sin embargo, no fue objeto de ninguna discusión por su parte: simplemente se dio por un hecho, probablemente considerado como obvio por el importante número de términos emparentados que con facilidad surgen al comparar las listas de vocabulario.

Posteriormente se postularon relaciones con otras lenguas. Por ejemplo, Cyrus Thomas (1902) incluyó una familia dorasque en su lista de familias lingüísticas integrándola con el cháliva, el chánguena, el chumulu, el gualaca, el dorasque y una lengua geográficamente distante: el rama de Nicaragua. Sin embargo, correspondió, hasta donde he podido determinar, a de La Grasserie (1904) el mérito de reconocer (sin demostrarla eso sí) la pertenencia del grupo dorasque-chánguena a la familia chibcha, cuya existencia había sido descubierta por Max Uhle dieciséis años antes.

Las clasificaciones que han venido después se pueden dividir en dos:

a) aquellas que no relacionan el grupo dorasquechánguena de manera especial con ninguna otra lengua ni ningún grupo mayor de lenguas dentro de la familia y

b) aquellas que consideran al dorasque-chánguena como subgrupo de alguna rama de la familia.

El segundo esquema ha sido el favorito de la mayor parte de quienes se han ocupado de la clasificación de las lenguas chibchas $y$, al parecer a partir de Rivet (1912), el dorasque y el chánguena han sido incluidos como subgrupo dentro de un grupo mayor que incluye al bocotá y el movere. Este grupo, con frecuencia denominado istmico, formaría parte según Jijón y Caamaño (1943), Mason (1950), Tax (1960) y otros, de una rama del Pacífico de la familia chibcha (cuyo otro integrante sería un grupo colombiano con lenguas como el lile, el timba, el yolo, etc.) y según Schmidt (1926, p. 213) de una rama noroeste o talamanca-dorasque de la familia (que abarcaría también a un grupo integrado por el guatuso y el rama, otro por el bribri, el cabécar, el térraba y el boruca y otro por el cuna).

Frente a las posiciones citadas, Loukotka (1942, 1968) mantuvo al dorasque-chánguena como rama independiente de la familia.

Conviene aclarar que las agrupaciones mencionadas no se han basado en discusiones explícitas, en trabajos publicados, de las razones para clasificar de uno u otro modo, sino que, hasta donde es posible comprobarlo, parecen reflejar simples opiniones de los diversos autores. La mayor parte de éstos no da ningún argumento ni elemento de juicio. Algunos incluyen en sus obras listas de vocabulario paralelas de las lenguas comparadas pero sin señalar cuáles palabras están emparentadas y cuáles no; otros, en particular, Beuchat y Rivet (1910) y Jijón y Caamaño intentan demostrar sus puntos de vista, pero su desconocimiento prácticamente total de los métodos de la lingüística diacrónica (véase Constenla, 1983, pp. 17-33) hizo inútiles sus esfuerzos.

\section{Propósito de este trabajo}

Como tal vez se haya podido colegir de lo anteriormente comentado, las interrogantes a las que es necesario responder en el caso de las lenguas o dialectos que nos ocupan son varias:

a) ¿Están relacionadas genéticamente?

b) ¿Pertenecen a la familia chibcha?

c) ¿Constituyen un grupo dentro de dicha familia?

ch) Si constituyen un grupo, ¿cómo se subagrupan dentro de él?

d) Si constituyen un grupo, ipertenece dicho grupo a su vez a una agrupación mayor dentro de la familia?

El propósito de este trabajo es contestar -tentativamente en algún caso, pero siempre con argumentaciones bien fundamentadas y explícitas-las preguntas planteadas.

2. Coincidencias léxicas y correspondencias fonéticas entre el chumulu, el gualaca, el dorace y el chánguena

Para determinar la relación entre las variedades que nos ocupan se compararon todos los rubros de idéntico significado que se pudieron conseguir en las cuatro hablas. En el caso del dorace, no se tomaron en cuenta los rubros traducidos por medio de frases o por formas de tipo onomatopéyico. Los hispanismos como kóci (chumulu, gualaca, chánguena) -cochí (dorace) 'cerdo' se eliminarán también. La lista de elementos comparados se da a continuación, señalando por medio del subrayado los términos no emparentados (la grafía usada es la de las fuentes): 


\begin{tabular}{|c|c|c|c|c|c|}
\hline & Castellano & chumulu & gualaca & dorace & chánguena \\
\hline 1 & agua & $\mathrm{ji}, \mathrm{si}$ & $\mathrm{ti}$ & yi & $\mathrm{ji}$ \\
\hline 2 & gavilán, águila & bu & bu & -bú & $\mathrm{bu}-\overline{\mathrm{o}}$ \\
\hline 3 & banco & sérkala & sérkala & sergual & sérkala \\
\hline 4 & boca & kagué & kaga & shur & semén \\
\hline 5 & bueno, bien & hapé & apé, hapé & apé & 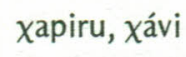 \\
\hline 6 & cabeza & dukú & kinúnúma & ducú & du \\
\hline 7 & cacao & doló & doló & dolol & doló \\
\hline 8 & canoa & ulú & ulú & ul & ulú \\
\hline 9 & casa & hu & hu & hu, uu & $\chi \mathrm{u}$ \\
\hline 10 & chicha & $\theta$ agá & $\theta$ agá & shisali & $m a$ \\
\hline 11 & Dios & bál $\theta$ a & bál $\theta \mathrm{a}$ & balsá & bál $\theta$ a \\
\hline 12 & estrella & uxúse & viu & ushú & haita \\
\hline 13 & fuego & kebu & kebu & queb & keu \\
\hline 14 & gallina & kógri & kókri & cocrí & kókri \\
\hline 15 & garrapata & kátú & kátú & cató & olia \\
\hline 16 & grande & ha-oci & ha-oci & soldomá & 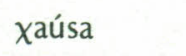 \\
\hline 17 & guacamayo colorado & kobá & kobá & cobal & vaiva \\
\hline 18 & hacha & olixá & olixá & xoligá & oliá \\
\hline 19 & hombre & taro & taro & tará & taro \\
\hline 20 & lagarto & dárki & daibike & dabquí & culi \\
\hline 21 & madre & to & to & toó & doló \\
\hline 22 & maíz & hábu & ábu & $a b$ & háu \\
\hline 23 & mano & kul-ósol & kalá & culgua & brais \\
\hline 24 & mazamorra & subá & $\theta$ ubá & shua & suvá \\
\hline 25 & mes, luna & siríla & sirála & shiril & sirála \\
\hline 26 & møsca & mulmūlu & mulmūlu & mormol & mulmūlu \\
\hline 27 & mujer & biá & wiá & segá & biá \\
\hline 28 & nariz & nekó & negú & nenco & $\theta a k a i$ \\
\hline 29 & ñame & tu & tu & saá & tu \\
\hline 30 & ojos & okó & okó & oc, ocó & ukú \\
\hline 31 & olla, tinaja & hu & ut, u & uu, u & hu, u \\
\hline 32 & oreja & kugá & kugá & ogausí & tayó \\
\hline 33 & padre & - su & $\theta \mathrm{u}$ & paá & kága \\
\hline 34 & perico & kosó & kosó & coshó & sorón \\
\hline 35 & perro & viló & viló & biló & viló \\
\hline 36 & pez & kisi & kisi & quishí & klun \\
\hline 37 & pie & ser & séro & sergua & séri \\
\hline 38 & piedra & hak, hagá & agá & ag & hagá \\
\hline 39 & piojo & $k \bar{u}$ & $k \bar{u}$ & cuú & $k \bar{u}$ \\
\hline 40 & pulga & bágue & bágue & сии́ & bágue \\
\hline 41 & saíno & biró & biró & biró & biró \\
\hline 42 & sal & báli & báli & balí & báli \\
\hline 43 & serpiente & búli & kankála & korgoshá & búli \\
\hline
\end{tabular}




\begin{tabular}{|c|c|c|c|c|}
\hline sol & kerél & kerele & kerel & kelíku \\
\hline tigre & ho- & $0-$ & o & cáli \\
\hline totuma, guacal & sok, $\theta$ ok & sok, $\theta$ ok & sooc & sok, $\theta$ ok \\
\hline uno & kúe & kúe & cumac & umai \\
\hline venado & karí & karí & calí & karí \\
\hline viento & xatú & xatú & batshalá & xatú \\
\hline yuca & ik & ígá & ic & hi \\
\hline
\end{tabular}

La observación de las listas anteriores, integradas por términos pertenecientes al vocabulario básico en su mayor parte, y obviamente emparentados también la mayoría de ellos, permite encontrar

$\begin{array}{lc}\text { Números de los rubros } & \text { chumulu } \\ 1,18,25,35,41,47 & \mathrm{i} \\ 2,6,9,24,31,39 & \mathrm{u} \\ 3,13,37,43 & \mathrm{e} \\ 5,11,18,19,22,38 & \mathrm{a} \\ 7,14,35,41,45 & \mathrm{o} \\ 13,14,30,39,43,45 & \mathrm{k} \\ 2,11,41,42 & \mathrm{~b} \\ 3,11,24,25,37,45 & \theta, \mathrm{s}\end{array}$

La respuesta a la primera pregunta es, pues, definitivamente que sí están emparentadas estas hablas, y que su parentesco es claramente bastante estrecho.

3. Pertenencia del chumulu, el gualaca, el dorace y el chánguena a la familia chibcha

En un estudio anterior (Constenla 1981, pp. 266-273) di pruebas de que en efecto estas hablas pertenecen a la familia chibcha. A continuación cito, a modo de ejemplo, ocho de los étimos protochibchas de los cuales se han hallado reflejos en ellas:

\section{*bur: 'ceniza'}

rama plun, guatuso puru:, cabécar bũlî́tu,

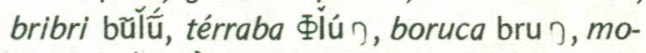
vere ๆúms岀, bocotá mnúgda, chumulu bulku, gualaca bulukú, dorace bulcú, tunebo burá, cuna puru, cágaba muli, guamaca bun, bintucua búnzga, atanques bun, chimila muúnha. rápidamente numerosas correspondencias fonéticas algunas de las cuales se señalan a continuación, con el número de algunos de los conjuntos de palabras emparentadas en que se presentan:

$\begin{array}{ccc}\text { gualaca } & \text { dorace } & \text { chánguena } \\ \mathrm{i} & \mathrm{i} & \mathrm{i} \\ \mathrm{u} & \mathrm{u} & \mathrm{u} \\ \mathrm{e} & \mathrm{e} & \mathrm{e} \\ \mathrm{a} & \mathrm{a} & \mathrm{a} \\ \mathrm{o} & \mathrm{o} & \mathrm{o} \\ \mathrm{k} & \mathrm{k} & \mathrm{k} \\ \mathrm{b} & \mathrm{b} & \mathrm{b} \\ \theta, \mathrm{s} & \mathrm{s}, \mathrm{sh} & \theta, \mathrm{s}\end{array}$

*apí: 'sangre'

rama árbi, cabécar pí, bribri ap $\hat{\imath}$, bocotá be, chumulu y gualaca havé, dorace ab, muisca yba, tunebo ába, cuna ape, cágaba abi, guamaca áma, dobocubí abiy.

*dù: 'ave'

cabécar dù, bribri dú, boruca ducít, movere núgu's, muisca sue, cuna nuu ('ave comestible'), cágaba nuba, chumulu dul.

*hulù: 'balsa, bote' rama út, cabécar hulùkaľ̀, bribri ulúkằ̀, boruca ru, térraba lú, movere rú, bocotá du, chumulu, gualaca y chánguena úlu, dorace ul, cuna ulu.

*u rú: 'cedro' (diversos árboles del género Cedrela) rama út, guatuso u $\Omega_{\mathrm{\eta}}$, bribri uluk, chumulu y gualaca ulú, cuna ulu, cágaba ululá.

*əร ə̀: 'niño, hijo, huevo' 
rama ắt 'huevo', guatuso ara:fa 'progenie', bribri alá 'niño, hijo, huevo', dorace alal, 'niño', tunebo aná 'huevo', cuna ana 'cría'.

*pú́: 'ave de rapiña' rama punkit, cabécar pṹ, bribri pṹ, térraba థú n, chumulu y gualaca bu, movere mú, bocotá mu, guamaca bu.

*kuhká kuhkuá 'oreja'

rama kukwa ūp, cabécar kuk@́, bribri kuk'́, boruca kuágax, chumulu y gualaca kugá, muisca cuhuca, tunebo kukahá, cágaba kuka, guamaca kuhkúa, bintucua kúkuë, atanques kukkuá.

Otros étimos observados incluyen los morfemas para agua, árbol, boca, casa, camarón, cola, cuatro, dormir, dos, hambre, lengua, leña, luna, llorar, mano, mono aullador, nube, pejivalle o pixbá, pecarí, pequeño, piedra, piel, piojo, raíz comestible (ñame, otoe), tabaco, tinaja, olla, tres, uno, viejo y yuca.

Por medio de este tipo de material se pudo constatar la evolución en chumulu, en gualaca, en dorace $y$, en menor grado, en chánguena de la mayor parte de los fonemas reconstruidos para la protolengua.

\section{El dorasque-chánguena como grupo dentro de} la familia chibcha

Para responder a la tercera pregunta planteada, se llevó a cabo una comparación con diecisiete de las otras dieciocho lenguas reconocidas en mi estudio de 1981 como pertenecientes a la familia chibcha. Para ello se disponía de una lista de 126 rubros de vocabulario básico (incluidos entre los que integran la lista glotocronológica de 215 rubros, véase Swadesh, 1955) en dichas lenguas que se había empleado en investigaciones precedentes (Constenla, 1984). De dicha lista se tomaron los rubros para los cuales se contaba con los equivalentes en chumulu, gualaca y chánguena, desechándose los demás. Esto la redujo a 25 rubros (24 en el caso de una de las lenguas comparadas: el chimila, para la que no se consiguió el equivalente de 'malo'). Esta lista con sus equivalentes en todas las lenguas comparadas se incluye en la tabla 1. En ella se han subrayado los términos de las lenguas emparentadas con formas chánguenas $\mathrm{y}$, se han marcado con \& los emparentados con formas tanto chumulus como gualacas y se han marcado con \# los emparentados sólo con formas gualacas.
Los porcentajes de términos emparentados (cuyo reconocimiento se basa en este trabajo en las correspondencias determinadas en Constenla, 1981), se incluyen en la tabla 2. En el caso del dorace, se obtuvieron sólo 22 de los rubros de la lista de 25 ('diente' y 'noche' se desecharon por haber sido traducidos por medio de frases del tipo improvisado comentado en 0.2.; 'malo' no aparece en los materiales disponibles). Los porcentajes de términos emparentados correspondientes a esta lengua se dan también en la tabla 2.

La observación de dichos porcentajes permite comprobar que el chumulu, el gualaca, el chánguena y el dorace tienen entre sí unos mucho más elevados (el más bajo es $55 \%$ ) que los que comparten con las otras lenguas (el más alto de los cuales es $36 \%)$. El porcentaje más elevado es el del chumulu con el gualaca (84\%). Aplicando los principios de Dyen (1962), el dorace y el chánguena se pueden agrupar con aquellas lenguas, pues ambos tienen sus porcentajes más elevados con el chumulu (dorace-chumulu: $73 \%$ y chánguena-chumulu: $64 \%$ ) y éstos no son significativamente diferentes (según la prueba de ji al cuadrado) del que tiene el chumulu con el gualaca. El porcentaje más elevado de una de estas cuatro hablas con otra lengua es el del chumulu con el movere $(36 \%)$, que sí es significativamente diferente del chumulu con el gualaca. Consecuentemente, el dorasque-chánguena constituye un grupo bien definido dentro de la familia chibcha.

Lo indicado por dichos porcentajes, se ve confirmado por numerosas innovaciones léxicas como, por ejemplo, xatú 'viento' en chumulu, gualaca y chánguena frente al étimo de la protolengua presente en rama, guatuso, movere, bocotá, cuna, cágaba, bintucua, atanques $y$, probablemente, en chimila (*bus -$)$; chumulu y dorace kerel, gualaca kerele y chánguena keliku 'sol' frente a étimos más extendidos en la familia como $* k a$, paya $a$, boruca kak, cabécar kãú́ y *dì, bribri díù̀, chimila n:ínga, dobocubí día, y chumulu, gualaca, chánguena doló, dorace dolol 'cacao' frente al étimo reflejado por el rama kúk, guatuso kaxu:, térraba kó, boruca káu?, movere ku, etc.

Otro indicio que favorece la agrupación es la fusión de *k y *g en $k$ (*kû́ $>$ kū 'piojo'; *gul-> kul- 'mano') fenómeno que no se da en ninguna de las lenguas circundantes (el guatuso es la única otra lengua de la familia que lo presenta, pero de modo completamente independiente como parte de la fusión de todas las oclusivas sonoras con las sordas). 
TABLA 1

\begin{tabular}{|c|c|c|c|c|c|c|c|c|c|}
\hline \multicolumn{2}{|c|}{ Castellano } & \multirow{2}{*}{$\begin{array}{l}\text { chumulu } \\
j / \&\end{array}$} & \multirow{2}{*}{$\begin{array}{l}\text { gualaca } \\
t / \&\end{array}$} & \multirow{2}{*}{$\begin{array}{l}\text { chánguena } \\
j i \text { \& }\end{array}$} & \multirow{2}{*}{$\begin{array}{l}\text { paya } \\
\text { asó }\end{array}$} & \multirow{2}{*}{$\begin{array}{l}\text { rama } \\
s i \&\end{array}$} & \multirow{2}{*}{$\begin{array}{l}\text { guatuso } \\
t i: \&\end{array}$} & \multirow{2}{*}{$\frac{\text { bribri }}{\text { dǐ? \& }}$} & \multirow{2}{*}{$\frac{\text { cabécar }}{\text { dik/ \& \& }}$} \\
\hline 1 & agua & & & & & & & & \\
\hline 2 & boca & kagué \& & kaga \& & semen & sapá & kaká \& & ko:ki \& & axkó \& & khó \& \\
\hline 3 & bueno & hapé \& & apé \& & xapiru \& & ainyá & báin & mara:la: arina: & buǎ & ból \\
\hline 4 & cabeza & dukú \& & kinúnúma & $d u \&$ & sa & kin & ča: & uQ̂kìd & çákù \\
\hline 5 & diente & $s u \&$ & $\theta u \&$ & $s u \&$ & ki-há & sik & oka: & aká & kà \\
\hline 6 & estrella & uxuse & viu \# & haita & piriwá & piúp \# & $4 \mathrm{i}:$ joki: & b'tku & bıkuó \# \\
\hline 7 & hombre & taro \& & taro \& & taro \& & arwá & nikíkna & oča:paka: & wib & hajijì \\
\hline 8 & luna & sirila\& & sirála \& & sirála \& & yauwi & túkan & $\$ i x i: \&$ & šù \& \& & tulù \\
\hline 9 & madre & to & to & doló & kaki & máma & su: & ãbí & bî́dã \\
\hline 10 & malo & irógla & irógla & guali & warki & kutkula & e:me koka:rina: & sulǔ & salui \\
\hline 11 & mano & kulósol \& & kalá & brais & sawá & kuík & kuik & ulá \& & hulà \& \\
\hline 12 & mujer & biá \& & wiá \& & biá \& & kortá & kumá & kuri:xuri: & ăâkò ľ & aláǩ́o \\
\hline 13 & nariz & nekó \& & negú \& & $\theta$ akai & tansá & táik & tai:ki & jik & Yík \\
\hline 14 & noche & be \& & be \& & be \& & kakalnyá & kíkik & tiú:rika: & dãlễuè & tauidã \\
\hline 15 & ojo & okó \& & -okó \& & ukú \& & wa & úp & $\Phi i \notin \mathrm{i}:$ & uóbalà & uóbalà \\
\hline 17 & pez & kisi & kisi & klun & wiśa & sálpaka & muqu: & díbã́ & díbã̀ \\
\hline 18 & pie & sero \& & ser \& & seri \& & tah-há & kát & कo:kiča: & kaló & kaló \\
\hline 19 & piedra & haga \& & agá \& & hagá \& & sa & nal ín & okta ra: \& & âk \& & hák \& \\
\hline 20 & piojo & $k u \bar{u} \&$ & $k \bar{u} \&$ & $k \bar{u} \&$ & $u \&$ & kuri \& & $k u:$ \& & $k \hat{\tilde{u}} \&$ & kṹ \& \\
\hline 21 & sal & báli \& & báli \& & báli \& & také & táuli & ti: karu: & daǰi & dajt \\
\hline 22 & serpiente & búli \& & kankála & oúli \& & ewis-ká & álbut & $\$$ ala:ki & tkabi & tkabi \\
\hline 23 & sol & kerél \& & keréle \& & keliku \& & á & núnik & to:xi & diuè & kắu’o \\
\hline 24 & uno & $-\mathrm{e} \&$ & - \& \& & umai & as & sáimin & ana:kača: & ět̂ \& & étká \& \\
\hline 25 & viento & xatú & Xatú & xatú & aunipiś-ká & púlkat & pura: & sĩũ̃? & sĩũá \\
\hline
\end{tabular}

TABLA 1 (continuación)

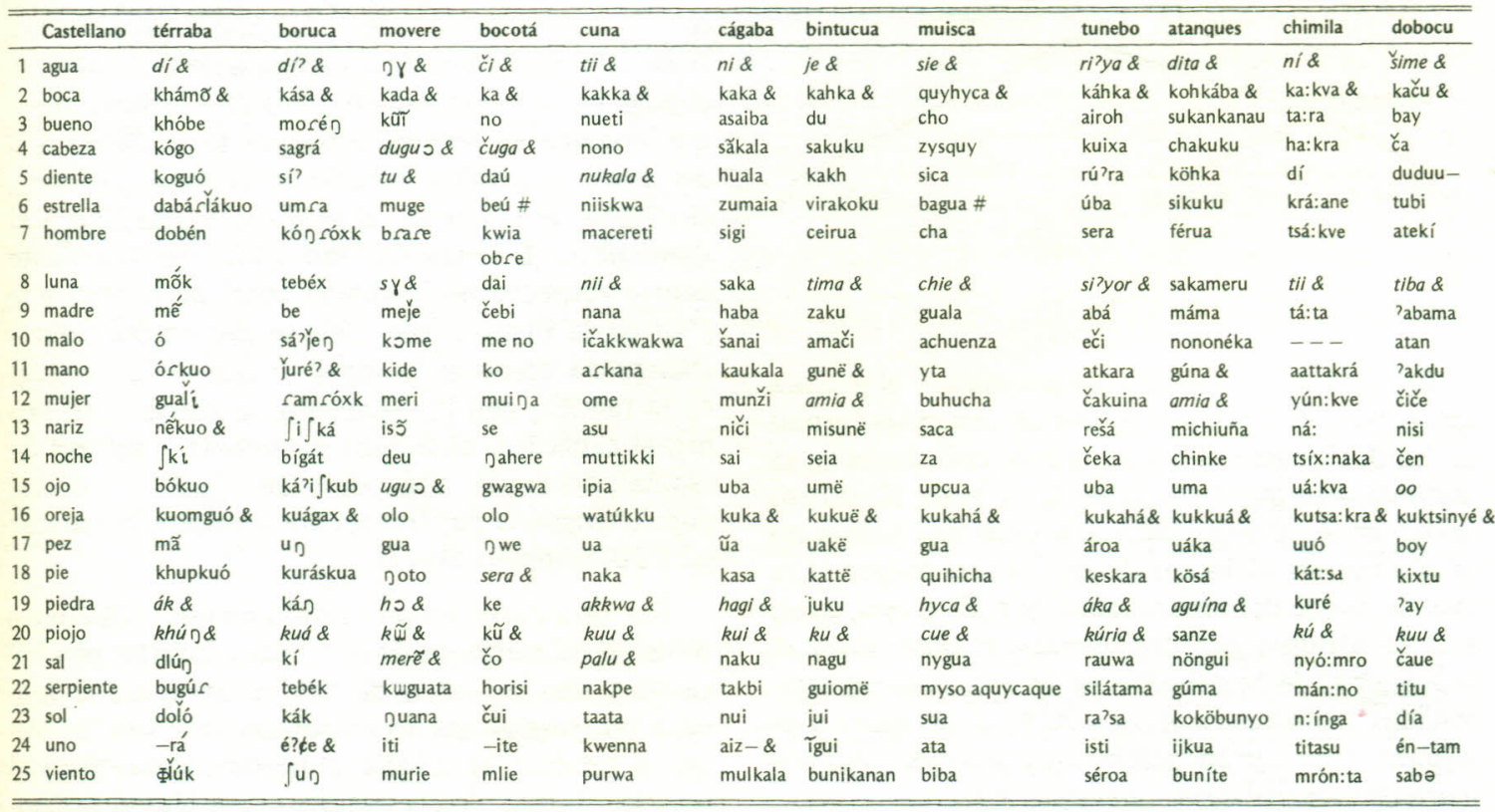


TABLA 2

PORCENTAJES DE TERMINOS EMPARENTADOS DEL CHUMULU, EL GUALACA, EL DORACE Y EL CHANGUENA ENTRE SI Y CON 17 LENGUAS CHIBCHAS

\begin{tabular}{|c|c|c|c|c|c|c|c|c|c|c|c|c|c|c|c|c|c|c|c|c|c|}
\hline & Chan & Chu & Gual & Dor & $\mathrm{Pa}$ & $\mathrm{Ra}$ & Guat & Bri & $\mathrm{Cab}$ & $\mathrm{Te}$ & Bo & Mo & Boc & $\mathrm{Cu}$ & Cag & Bin & $\mathrm{Mu}$ & Tu & At & Chi & Dob \\
\hline chánguena & - & 64 & 56 & 55 & 8 & 8 & 16 & 16 & 12 & 12 & 8 & 32 & 16 & 24 & 12 & 16 & 16 & 16 & 12 & 13 & 16 \\
\hline chumulu & 64 & - & 84 & 73 & 4 & 16 & 20 & 32 & 28 & 24 & 24 & 36 & 20 & 28 & 24 & 28 & 24 & 24 & 24 & 21 & 24 \\
\hline gualaca & 56 & 84 & - & 59 & 4 & 20 & 20 & 32 & 28 & 24 & 20 & 32 & 20 & 28 & 24 & 24 & 28 & 24 & 20 & 20 & 24 \\
\hline dorace & 55 & 73 & 59 & - & 5 & 9 & 18 & 23 & 18 & 14 & 14 & 32 & 18 & 23 & 14 & 18 & 18 & 18 & 14 & 14 & 18 \\
\hline
\end{tabular}

\section{Subagrupación interna del dorasque-chánguena}

Los porcentajes de la tabla 2 indican que las dos variedades más cercanas son el chumulu y el gualaca con un $84 \%$ de términos emparentados, en tanto el chánguena presenta con ambos, porcentajes mucho inferiores: $64 \%$ y $56 \%$ respectivamente. El dorace tiene un porcentaje bastante al to con el chumulu: $73 \%$, pero su porcentaje con el gualaca, $59 \%$ no es mucho más elevado que el que tiene con el chánguena, $55 \%$. Mi hipótesis al respecto es que el dorace representa la continuación del chumulu, sólo que sometido al cambio acelerado propio de los procesos de muerte de lenguas. Esta idea se ve confirmada por el hecho de que la separación que habría sufrido de su estado anterior: $27 \%=$ $100 \%-73 \%$, es casi la misma que media entre el porcentaje compartido por su estado anterior y el gualaca y el compartido por su estado más reciente $y$ el gualaca: $29 \%=84 \%-59 \%$.

El árbol genealógico resultante es, pues:

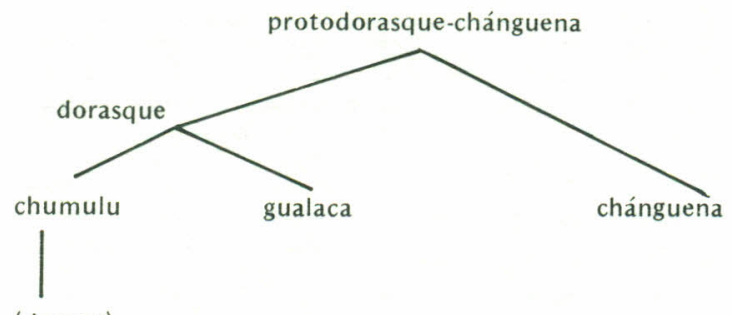

(dorace)

En vista de lo cercano del chumulu y el gualaca (en el total de los casos en que se tienen las formas de las dos lenguas el porcentaje de coincidencias es aún más alto que en la comparación de 50 rubros hecha en 2., parece claro que estas dos hablas no eran sino dos dialectos de una misma lengua, a la cual se puede denominar dorasque. El grupo, pues, está constituido por dos lenguas, el dorasque y el chánguena, de la primera de las cuales se registraron dos dialectos en el siglo XIX, el chumulu y el gualaca, y la continuación (muy alterada) del primero de esos dialectos en el siglo XX.

\section{Posibilidad de agrupar el dorasque-chánguena con otras lenguas dentro de la familia}

Desde el punto de vista del método comparativo, no hay indicios seguros que permitan subagrupar al dorasque-chánguena de manera clara con otras lenguas o grupos de lenguas, ni siquiera en el caso de aquellas que presentan un mayor grado de coincidencia con él en materia de léxico.

Por una parte, en tanto parece compartir con el bribri y el cabécar principalmente formas heredadas de la protolengua, como por ejemplo la raíz 'mano' ( $k u l$ - en chumulu y dorace, presente en atanques, bíntucua, boruca, bribri y cabécar) o la del numeral 'uno' ( $-e$ en chumulu y gualaca, presente en bribri, cabécar, boruca y cágaba) con el movere, el bocotá y el cuna comparte formas que son muy posiblemente innovadoras, como la raíz 'diente', su, presente en movere y cuna (y opuesta a la que se da en bribri, cabécar, guatuso, térraba y, quizás, en bíntucua y atanques); la raíz 'sal', bal-, presente en movere y en cuna (opuesta tanto a la que se da en bribri, cabécar y paya como a la de la mayor parte de las lenguas de Colombia incluidas en la comparación); y la raíz 'cabeza' du-, que aparece en movere y bocotá (y se opone a la del paya, el guatuso, el cabécar, el boruca, el cuna, el cágaba, el bíntucua, el muisca, el atanques y el dobocubí). En materia fonológica, algo semejante ocurre respecto de la fusión total de la $/ * \mathrm{p} /$ y la /*b/ de la protolengua, que se dio en dorasquechánguena como en la mayor parte de las lenguas de la familia, con la excepción, al parecer, del bribri, el cabécar y el térraba solamente: *pũ 'ave de rapiña', dorasque-chánguena bu; *bá? 'tú', dorasque-chánguena $b a$; frente, por ejemplo, al cabécar p $\widetilde{u}$ y bá, respectivamente.

Por otra parte, en un cambio menos atribuible a difusión, la metátesis de la ${ }^{*} u$ situada entre una fricativa y una secuencia de ${ }^{*} k V$, el dorasque se opone a las lenguas que lo presentan, que son el bribri, el cabécar, el térraba, el boruca, el movere y el bocotá. *huká 'piel' evolucionó a chumulu ugá, 
muisca huca, cuna ukka, bribri axkuǿ, térraba kuóta, boruca kuá's, movere kuáta, bocotá kuara (las últimas cuatro lenguas presentan formas en que se han añadido sufijos derivativos a la raíz).

Por lo que respecta a porcentajes de léxico compartido, los de la tabla 2 nos muestran una relación algo más estrecha con el movere y el cuna (en especial con la primera) por una parte, y con el bribri y el cabécar por otra. Es interesante señalar que los porcentajes casi en todos los casos son más bajos con el chánguena que con los dialectos del dorasque y que esto es especialmente notorio en el caso del bribri y del cabécar, en que las diferencias son muy pronunciadas. El porcentaje del bribri con el chumulu o el gualaca, por ejemplo, es el doble del que tiene la misma lengua con el chánguena. El movere, en cambio, muestra el mismo porcentaje con el chánguena y el gualaca. En el caso del cuna la diferencia entre el porcentaje comparti- do con el chánguena ( $24 \%$ ) y el compartido con los dialectos del dorasque $(28 \%)$ es pequeña. Esto podría hacer pensar en una relación algo más estrecha con el movere y el cuna, pero la comparación de una muestra más amplia (del dorasque -chumulu/dorace - con 12 lenguas) cuyos porcentajes se dan en la tabla 3 , indica que desde el punto de vista léxico, si se aplican los criterios precisos establecidos por Dyen (1962), el dorasque no se subagrupa con ninguna de las otras lenguas de la familia incluidas. Según esta tabla la familia está constituida por la coordinación de una serie de lenguas aisladas (entre las que se cuenta el dorasque) y dos subgrupos (el constituido por el cabécar y el bribri, y el constituido por el cágaba y el bíntucua). La familia, a su vez, se relaciona con el paya, que no pertenece a ella, sin embargo, como lo indican los porcentajes (a este respecto véase Constenla, 1985).

TABLA 3

\begin{tabular}{|c|c|c|c|c|c|c|c|c|c|c|c|c|c|}
\hline & paya & rama & guatuso & bribri & cabécar & térraba & boruca & move & bocotá & cuna & cágaba & bíntucua & dorasque \\
\hline $\mathrm{Pa}$. & - & 14,5 & 10,8 & 16,9 & 19,3 & 8,4 & 10,8 & 8,4 & 12,0 & 8,4 & 10,8 & 12,0 & 8,4 \\
\hline $\mathrm{Ra}$ & 14,5 & - & 18,1 & 25,3 & 26,5 & 16,9 & 16,9 & 14,5 & 21,7 & 20,5 & 24,1 & 21,7 & 18,1 \\
\hline Guat. & 10,8 & 18,1 & - & 21,7 & 20,5 & 14,5 & 14,5 & 15,7 & 16,9 & 19,3 & 20,5 & 21,7 & 15,7 \\
\hline Brib. & 16,9 & 25,3 & 21,7 & - & 74,7 & 22,9 & 25,3 & 24,1 & 25,3 & 18,1 & 26,5 & 24,1 & 24,1 \\
\hline Cab. & 19,3 & 26,5 & 20,5 & 74,7 & - & 22,9 & 28,9 & 22,9 & 27,7 & 16,9 & 28,9 & 26,5 & 21,7 \\
\hline Te. & 8,4 & 16,9 & 14,5 & 22,9 & 22,9 & - & 19,3 & 19,3 & 16,9 & 15,7 & 19,3 & 20,5 & 16,9 \\
\hline Bor. & 10,8 & 16,9 & 14,5 & 25,3 & 28,9 & 19,3 & - & 19,3 & 19,3 & 16,9 & 20,5 & 19,3 & 20,5 \\
\hline Mov. & 8,4 & 14,5 & 15,7 & 24,1 & 22,9 & 19,3 & 19,3 & - & 32,5 & 20,5 & 25,3 & 22,9 & 22,9 \\
\hline Boc. & 12,0 & 21,7 & 16,9 & 25,3 & 27,7 & 16,9 & 19,3 & 32,5 & - & 19,3 & 26,5 & 26,5 & 19,3 \\
\hline Cu. & 8,4 & 20,5 & 19,3 & 18,1 & 16,9 & 15,7 & 16,9 & 20,5 & 19,3 & - & 20,5 & 20,5 & 22,9 \\
\hline Cág. & 10,8 & 24,1 & 20,5 & 26,5 & 28,9 & 19,3 & 20,5 & 25,3 & 26,5 & 20,5 & - & 42,2 & 21,7 \\
\hline Bint. & 12,0 & 21,7 & 21,7 & 24,1 & 26,5 & 20,5 & 19,3 & 22,9 & 26,5 & 20,5 & 42,2 & - & 20,5 \\
\hline Dor. & 8,4 & 18,1 & 15,7 & 24,1 & 21,7 & 16,9 & 20,5 & 22,9 & 19,3 & 22,9 & 21,7 & 20,5 & - \\
\hline
\end{tabular}

\section{Conclusiones}

En el presente estudio se ha comprobado con claridad la relación genealógica entre el chumulu, el gualaca, el dorace y el chánguena, su pertenencia a la familia chibcha y su condición de grupo bien definido dentro de dicha familia con una subagrupación interna en dos lenguas: el chánguena y el dorasque, dividida ésta última en dos dialectos (el gualaca y el chumulu-dorace). Por lo que atañe a 
la mayor o menor estrechez de las relaciones del dorasque chánguena dentro de la familia, las únicas indicaciones claras vienen de la comparación léxica, que favorece la posición de Loukotka según la cual el dorasque-chánguena es una rama independiente de la familia.

En cuanto a futuras investigaciones sobre estas lenguas, las prioridades a mi parecer deben ser:

a) Interpretar los materiales disponibles fonológicamente del mejor modo posible, de modo que se pueda uniformar su escritura con una transcripción de tipo fonológico: hay que determinar, por ejemplo, la relación de $s, s h, c h, j, y, \chi$ que aparecen en al ternancia en la escritura de algunos rubros.

b) Segmentar apropiadamente los materiales desde el punto de vista morfológico y sintáctico y realizar las identificaciones semánticas apropiadas. Por ejemplo, baxiktak glosado como 'sentarse' y 'toma asiento', está integrado por el pronombre ba 'tú' y xiktak 'sentarse'; ¿wa pesuk? '¿cómo estás?' es, probablemente, ¿ba apé suk?, literalmente '¿tú bien estás?'.

c) Con base en lo anterior, elaborar un diccionario en el que se incorporen todos los materiales del dorasque y disponerlos en los dos órdenes: castellano-dorasque, dorasque-castellano.

ch) Reconstruir con el mayor detalle posible el dorasque-chánguena.

d) Realizar comparaciones de listas de vocabulario lo más extensas que se pueda con las correspondientes de otras lenguas chibchas, con el fin de aclarar más el problema de la estrechez del parentesco con cada una de ellas.

\section{BIBLIOGRAFIA}

Beuchat, Henri y Paul Rivet. 1910. "Affinités des langues du Sud de la Colombie et du Nord de L'Equateur". Le Mouséon: 11:33-68, 141-98.

Constenla Umaña, Adolfo. 1981. "Comparative Chibchan Phonology". Tesis doctoral. Universidad de Pensilvania.

1983. "Desarrollo del estudio diacrónico de las lenguas chibchas (1888-1980)".
En: Estudios de Lingüística Chibcha // por M. Bertoglia, A. Constenla y E. Margery. Departamento de Publicaciones de la Universidad de Costa Rica.

1984. "El hüetar: observaciones sobre los materiales disponibles para su estudio y sobre las hipótesis en torno a sus afinidades lingüísticas". Revista de Filología y Lingüística de la Universidad de Costa Rica, 10 (2): 3-18.

1985. "Clasificación lexicoestadística de las lenguas de la familia chibcha". En: Estudios de lingüística chibcha IV, compilados por Adolfo Constenla y Enrique Margery. Editorial de la Universidad de Costa Rica, San José.

Dyen, Isadore. 1962. "The lexicostatistically determined relationship of a language group". In ternational Journal of American Linguistics, XXVIII (3), pp. 153-161.

Jijón y Caamaño, Jacinto. 1943. El Ecuador Interandino y Occidental, Vol. 3: Las lenguas del Sur de Centro-América y el norte y centro del Oeste de Sud-América. Quito: Editorial Ecuatoriana.

La Grasserie, Raoul de. 1904. "Les langues de Costa Rica et les idiomes aparentés". Journal de la Société des Américanistes de Paris, n. s., $1: 153-87$.

Loukotka, Cestmir. 1942. "Klassifikation der südamerikanischen Sprachen". Zeitschrift für Ethnologie 74: 1-69.

1968. Classification of South American Indian Languages. Publicada por Johannes Wilbert. Los Angeles: Latin American Studies Center, The University of California at Los Angeles.

Mason, John Alden. 1950. "The languages of South American Indians". En: J. H. Steward (comp.) Handbook of South American Indians 6: 157-317.

Miranda de Cabal, Beatriz. 1974. Un pueblo visto a través de su lenguaje. Panamá: Impresora $\mathrm{Pa}$ namá, S. A.

Pinart, Alphonse Louis. 1890. Vocabulario CasteIIano-Dorasque: dialectos Chumulu, Gualaca y 
Chánguina. Petite Bibliothéque Américaine II. Paris: Imprimerie de Destenay.

Rivet, Paul. 1912. Les familles linguistiques $d u$ Nord-Ouest de l'Amérique du Sud. Extrait de "L'Année Linguistique". Paris: Librairie C. Klincksieck.

Schmidt, Wilhelm. 1926. Die Sprachfamilien und Sprachen-Kreise der Erde. Heidelberg: Winter.

Swadesh, Morris. 1955. "Towards greater accuracy in lexicostatistic dating". Internacional Journal of American Linguistics 21:121-37.

Tax, Sol. 1960. "Aboriginal languages of Latin America". Current Anthropology, 1 (5-6): 431-6.
Thomas, Cyrus. 1902. "Provisional List of Linguistic Familles, Languages and Dialects of Mexico and Central America". American Anthropologist 4:207-16.

Torres de Araúz, Reina. 1980. Panamá indígena. Panamá: Instituto Nacional de Cultura.

Voegelin, C. F. y F. M. Voegelin. 1965. "Languages of the world: native America, fascicle 2". Anthropological Linguistics 7 (7). 
